\title{
Anomalies chromosomiques dans les gamètes humains
}

\author{
B Sèle, F Pellestor, S Rousseaux, L Raymond, \\ J Cozzi, R Pelletier
}

\author{
Laboratoire de Cytogénétique et Biologie de la Reproduction, Faculté de Médecine de Grenoble, \\ 38706 La Tronche Cedex, France
}

(28 Réunion de la Société Française pour l'Étude de la fertilité; Paris, 19-21 octobre 1989)

\begin{abstract}
Résumé - Chez l'homme, les nouveau-nés porteurs d'anomalies chromosomiques sont les survivants d'une très large cohorte de conceptions anormales. Une étude cytogénétique directe impliquerait leur destruction. De plus, la possibilité de déterminer l'origine parentale d'un chromosome surnuméraire ou manquant est limitée pour des raisons méthodologiques. L'étude cytogénétique des gamètes humains permet d'apporter des informations nouvelles concernant ces mécanismes. Plusieurs études portant sur de vastes échantillons de caryotypes spermatiques d'hommes normaux donnent un taux d'anomalies de $10 \%$. Nos résultats concernant 4 porteurs de translocations réciproques semblent montrer qu'il n'existerait aucune sélection contre les spermes anormaux. Puisque environ $30 \%$ des ovocytes inséminés dans le cadre d'un programme de FIV ne sont pas fécondés, les ovocytes humains sont devenus accessibles pour des études cytogénétiques à une large échelle. L'analyse d'ovocytes en métaphase II apporte des informations sur le taux de non disjonctions survenant au cours de la $1^{r e}$ division méiotique. Dans notre série de 405 caryotypes, le taux d'aneuploïdies est de $27 \%$. II n'existe aucune relation entre ce taux et le mode de stimulation de l'ovulation. Aucune augmentation liée à l'âge maternel n'a été observée.
\end{abstract}

\section{caryotype / spermatozoïdes / ovocytes / homme}

Summary - Chromosomal abnormallties in human gametes. Human newborns carrying chromosomal abnormalities are the survivors of a considerably larger cohort of affected conceptions. A direct cytogenetic study of these conceptuses would imply their destruction. Moreover, the ability to determine the parental origin of an additional or missing chromosome is limited from a methodological point of view. The cytogenetic study of human gametes provides information on these mechanisms. Several large-scale studies on sperm complements of normal men give an estimated abnormality rate of $10 \%$. Our results concerning four carriers of reciprocal translocations show no evidence for a selection against abnormal sperm. Since about $30 \%$ of the recovered eggs fail to become fertilized in IVF programs, human oocytes have become available for large cytogenetic studies. Analysis of oocytes II provides information on the first meiotic non-disjunction rate. In our sample of 405 karyotypes, the rate of aneuploidy was $27 \%$. No relationship was established between this frequency and the mode of stimulation of ovulation. No increase was observed with maternal aging.

karyotype / spermatozoa / oocytes / human 


\section{LA MUTAGÉNĖSE CHROMOSOMIQUE DANS L'ESPECE HUMAINE}

Par comparaison avec les autres mammifères, l'espèce humaine présente un taux de mutation chromosomique élevé. L'incidence des anomalies chromosomiques à la naissance est considérablement minorée du fait de mécanismes de sélection intervenant dès la période péri-implantatoire puis tout au long de la gestation. Pour en apprécier directement l'importance, il faudrait pouvoir établir un grand nombre de caryotypes d'ceufs humains fécondés. Or la technique cytogénétique est une méthode invasive d'exploration. En pratique elle suppose la destruction de l'œuf fécondé, car l'analyse de ses blastomères après biopsie reste encore du domaine de la spéculation. Dès lors on comprend qu'il n'existe encore à l'heure actuelle aucune étude épidémiologique de l'œư humain fécondé.

Les anomalies du nombre de chromosomes trouvent leur source dans le processus de non disjonction (ou malségrégation) méiotique. Pour en étudier la nature et la fréquence, on doit établir les caryotypes des cellules ayant franchi les méioses I ou II. L'étude de la méiose I n'apporte aucune information sur ce plan puisque le processus de ségrégation aléatoire des chromosomes n'a pas encore eu lieu. L'étude de la méiose II ne permet de comptabiliser que les non disjonctions qui se sont produites au cours de la méiose 1 . Seule l'étude des gamètes mûrs offre la possibilité de faire le décompte des accidents des deux méioses cumulées. Elle permet aussi une distinction entre les anomalies d'origine paternelle et celles d'origine maternelle.

\section{LES LIMITES DE L'INTERPRÉTATION CYTOGÉNÉTIQUE}

Lorsque l'on veut déterminer le caryotype somatique d'un individu, on observe les chromosomes de plusieurs de ses cellules (voire plusieurs dizaines) provenant éventuellement de tissus différents et l'on ne retient que l'anomalie qui se rencontre statistiquement le plus souvent, car toutes ces cellules sont théoriquement identiques (exception faite des rares cas de mosaïques). Ce procédé conduit donc à éliminer les anomalies qui ne se rencontrent que dans une seule cellule, essentiellement des hypodiploïdies, que l'on ne peut pas distinguer de pertes artéfactuelles et dont la signification est de ce fait discutable. En revanche, cette démarche n'est pas applicable au caryotype gamétique car chaque gamète est le produit unique de la mécanique méiotique et présente donc un caryotype qui lui est propre. De ce fait, il existe une marge d'incertitude plus grande dans son interprétation.

Le caryotype méiotique fût pendant longtemps la seule source d'information sur la cytogénétique de la reproduction. Celui-ci est établi par examen direct de coupes histologiques des gonades. L'aspect des chromosomes méiotiques est totalement différent de celui des chromosomes somatiques. A la métaphase I ou II, leur degré de condensation est trop important pour que leur soient appliquées des techniques de diagramme de bande, d'où le caractère rudimentaire de l'information que l'on peut en tirer. Par ailleurs, l'obligation de procéder à une analyse directe sans passer par une culture fait que les données ainsi recueillies sont de nature ponctuelle et ne permettent pas d'analyse statistique. 


\section{LE CARYOTYPE OVOCYTAIRE}

Les données cytogénétiques relatives au gamète femelle humain ont jusqu'ici été très limitées. L'observation des chromosomes méiotiques sur des biopsies d'ovaire fotal ou adulte n'apporte aucun renseignement en matière de mutagénèse chromosomique puisque tous les ovocytes contenus dans l'ovaire sont bloqués en prophase de méiose I. Par contre des travaux déjà anciens (Jagiello et al, 1968) ont apporté indirectement un début de contribution à la connaissance du gamète femelle. Ces travaux ont été basés sur l'observation de métaphases de méiose I et II d'ovocytes humains préovulatoires recueillis au cours d'une laparotomie, à la suite d'un traitement d'induction comportant HMG-HCG, chez des patientes volontaires. Bien entendu les données ainsi recueillies étaient des plus limitées en nombre. C'est cependant la même source d'information qui s'est trouvée subitement alimentée de façon massive par l'application en clinique humaine de la FIV. En effet
30 à $40 \%$ des ovocytes préovulatoires recueillis font l'objet d'un échec de FIV et sont légitimement utilisables pour l'analyse cytogénétique. Bien que le rendement, en terme de caryotypes interprétables, soit faible, la quantité de matériau disponible supplée à ce déficit.

Dans une minorité de cas, on observe des caryotypes diploödes. Ils ont été parfois interprétés par certains auteurs comme des métaphases II diploïdes ou même des endoreduplications, alors qu'il pourrait s'agir, beaucoup plus simplement, de stades diacinèse c'est-à-dire de prométaphases I parfaitement normales en rapport avec le caractère immature des ovocytes considérés.

La majorité des caryotypes établis proviennent de métaphases II. Ce sont ces stades qui offrent le plus d'intérêt, car le nombre de chromosomes résulte de leur ségrégation lors de la division précédente, c'est-à-dire de la méiose I.

La survenue d'une malségrégation se traduit par un défaut ou un excès de chromosomes, et statistiquement on attend au-

Tableau I. Etudes cytogénétiques des ovocytes II humains.

\begin{tabular}{lllllll}
\hline & $\begin{array}{l}\text { Veiga } \\
1987\end{array}$ & $\begin{array}{c}\text { Plachot } \\
1988\end{array}$ & $\begin{array}{c}\text { Bongso } \\
1988\end{array}$ & $\begin{array}{c}\text { Van Blerkom } \\
1988\end{array}$ & $\begin{array}{c}\text { Djalali } \\
1988\end{array}$ & $\begin{array}{c}\text { Sèle } \\
1989\end{array}$ \\
\hline $\begin{array}{l}\text { Métaphases II } \\
\text { interprétables }\end{array}$ & 117 & 316 & 251 & 135 & 96 & 377 \\
Hypohaploïdies & 3 & $?$ & 33 & 9 & 24 & 55 \\
& $(25 \%)$ & - & $(62 \%)$ & $(82 \%)$ & $(92 \%)$ & $(54 \%)$ \\
Hyperhaploïdies & 9 & 38 & 20 & 2 & 2 & 47 \\
& $(75 \%)$ & - & $(38 \%)$ & $(18 \%)$ & $(8 \%)$ & $(46 \%)$ \\
Aneuploïdies & 11 & $76 *$ & 53 & 11 & 26 & 102 \\
& $(9 \%)$ & $(24 \%)$ & $(21 \%)$ & $(8 \%)$ & $(27 \%)$ & $(27 \%)$ \\
\hline
\end{tabular}

- Le taux d'aneuploïdie a été estimé par doublement du nombre d'hyperhaploïdies. 
tant d'anomalies par excès que par défaut. De la comparaison des résultats publiés (tableau I), il ressort d'importantes discordances dans la proportion des deux catégories d'anomalies, ce qui est à imputer au caractère approximatif des caryotypes d'ovocytes II. Le plus souvent, les anomalies par défaut prédominent. Ceci peut s'interpréter de deux façons : le plus vraisemblable est qu'il s'agit de pertes d'ordre technique, mais il est plausible aussi que ces hypoploïdies résultent non pas de malségrégations, mais d'un autre mécanisme, à savoir la perte anaphasique de chromosome, qui ne comporte aucune contrepartie dans l'autre cellule fille. Ce caractère approximatif se traduit a fortiori au niveau du taux global d'anomalies, qui varie de 8 à $27 \%$. L'importance de l'écart type n'autorise pas la recherche de variations interindividuelles, d'autant que les effectifs par patiente sont toujours faibles.

Si l'on estime devoir retenir un taux général de l'ordre de $25 \%$ se pose encore la question du caractère très particulier des échantillons analysés, car tous ces ovocytes ont fait l'objet d'un échec de fécondation. C'est là que peut être mise à profit I'hétérogénéité des échantillons. En effet, on peut distinguer deux situations d'échec de fécondation selon l'origine présumée de cet échec (cause spermatique ou inex- pliquée) (tableau II). II n'existe pas de différence des taux d'anomalie, contrairement à ce qui avait été suspecté lorsque l'échantillon analysé était de plus petite taille (188 métaphases II au lieu de 377) (Pellestor et Sèle, 1988). On peut donc considérer, avec les réserves dues au caractère approximatif de cette approche, qu'une anomalie chromosomique ne serait pas un obstacle à la fécondabilité de l'ovocyte qui la porte. Un autre caractère particulier aux échantillons est lié au fait que ces ovocytes sont recueillis à la suite de protocoles d'induction de l'ovulation. Ces protocoles peuvent schématiquement être distingués selon 3 classes (tableau III). On n'observe pas de différence significative. Chez la plupart des mammifères, la stimulation ovarienne n'augmente pas la production d'anomalies chromosomiques. $\mathrm{Ce}$ n'est que chez une espèce particulière de hamster qu'il existerait d'après Hansmann (voir discussion Veiga et al, 1987) un effet corrélé à la dose. Dans l'espèce humaine, cette corrélation n'est pas établie (tableau IV).

L'influence de l'âge maternel sur la survenue d'anomalies chromosomiques est bien connu. Il est très marqué dans le cas particulier de la trisomie 21 au moment de la naissance, mais il existe aussi pour de nombreuses autres trisomies, comme le démontre l'analyse de produits d'avorte-

Tableau II. Taux d'aneuploïdie en fonction de l'origine présumée de l'échec de fécondation.

$\begin{array}{llr}\text { Cause spermatique } & 123 & 29(24 \%) \\ \text { Echec inexpliqué } & 254 & 73(29 \%) \text { * } \\ & 377 & 102(27 \%)\end{array}$

\footnotetext{
* Ecart non significatif $(P>0,05)$.
} 
Tableau III. Taux d'aneuploïdie en fonction du protocole d'induction.

Métaphases "I

interprétables
Aneuploidies

115

$28(24 \%)$

113

$44(33 \%)$

50

$12(24 \%)$

$84(28 \%)$

Ecart non significatif $(P>0,05)$.

ments spontanés (Hassold et Chiu, 1985). Le plus généralement, on admet que cet effet est dû à une corrélation entre l'âge maternel et la production d'ovocytes anormaux, car la majorité de ces anomalies est effectivement d'origine maternelle. II se trouve de surcroît que ces anomalies résultent le plus souvent de malségrégations en méiose I. Ces échantillons d'ovocytes II représentent un matériau de choix pour vérifier ces hypothèses (Sèle et al, 1987). Les résultats sont curieusement inverses de ceux attendus (tableau V).
Ces résultats sont à prendre sous toute réserve, car plusieurs facteurs en limitent la portée : 1) nous ne disposons que de très peu de données chez les patientes de plus de 40 ans; 2) il s'agit de toutes les anomalies confondues alors qu'une étude portant exclusivement sur les nullo et disomies 21 par exemple nécessiterait un échantillon de plusieurs milliers d'ovocytes; 3) les variations des taux globaux rapportés par les différentes équipes sont d'un ordre de grandeur supérieur à celui de l'effet recherché.

Tableau IV. Taux d'aneuploïdie en fonction de la dose d'HMG.

\begin{tabular}{lcr}
\hline Ampoules & $\begin{array}{c}\text { Métaphases II } \\
\text { interprétables }\end{array}$ & Aneuplö̈dies \\
\hline $0-12$ & 39 & $10(26 \%)$ \\
$13-23$ & 42 & $11(26 \%)$ \\
$24-65$ & 41 & $8(20 \%)$ \\
& 122 & $29(24 \%)$ \\
\hline
\end{tabular}

Ecart non significatif $(P>0,05)$. 
Tableau V. Taux d'aneuploïdie en fonction de l'âge.

\begin{tabular}{lcc}
\hline & $\begin{array}{c}\text { Métaphases II } \\
\text { interprétables }\end{array}$ & Aneuploïdies \\
\hline $22-28$ ans & 74 & $27(36 \%)$ \\
$29-34$ ans & 184 & $40(22 \%)$ \\
$35-40$ ans & 80 & $17(21 \%)$ \\
& 338 & $84(25 \%)$ \\
\hline
\end{tabular}

Ecart non significatif $(P>0,05)$.

\section{LE CARYOTYPE DU SPERMATOZOIDDE}

Si le gamète mâle comporte également 23 chromosomes, la comparaison avec le gamète femelle sur le plan cytogénétique s'arrête là, car le contexte est totalement différent : sa production en grand nombre autorise une analyse à faible rendement; le noyau dont la condensation est extrême ne voit ses chromosomes s'individualiser que lors de la fécondation; chaque métaphase analysée correspond de ce fait à la première mitose de segmentation, et le corollaire, c'est que l'on observe des chromosomes mitotiques bien décondensés et accessibles aux techniques de diagramme de bandes. Les données ainsi recueillies sont donc incomparablement plus précises. On notera au passage une particularité propre aux premières étapes du développement chez tous les mammifères : la décondensation des hétérochromatines constitutives, centromériques en particulier.

Pour éviter de détruire des œufs humains fécondés, on a recours à des fécondations hétérospécifiques, qui ne sont possibles qu'avec l'ovocyte de hamster doré. La technique est délicate (Sèle et al, 1985). On peut au mieux espérer un rendement de l'ordre de 9 caryotypes inter- prétables pour 100 ovocytes de hamster inséminés (Pellestor et Sèle, 1989).

Tous les travaux sont concordants pour estimer à $10 \%$ chez le sujet normal, la proportion de spermatozoïdes anormaux. Ceci n'est pas à comparer aux $25 \%$ du gamète femelle, car ce taux correspond au cumul des malségrégations de méiose I + II.

Les études de porteurs de translocations apportent la preuve qu'un contenu génétique anormal n'est pas un obstacle au pouvoir fécondant d'un spermatozoïde, du moins dans ce système qui n'implique pas de traversée de la pellucide; on peut observer jusqu'à $80 \%$ de caryotypes spermatiques anormaux.

L'apport de cette méthode est fondamental pour l'analyse de ségrégation des translocations. En effet, dans l'espèce humaine l'analyse de ségrégation est d'une façon générale très limitée du fait de la taille réduite des fratries. Or ce procédé consiste à obtenir un nombre élevé de fécondations pour un seul sujet, ce qui revient à simuler, sur le plan cytogénétique, une fratrie de très grande taille.

Prenons l'exemple de la translocation 13;14, connue pour être peu dargereuse pour la descendance des hommes fertiles qui en sont porteurs. De fait, nombreux 
sont les spermatozoïdes normaux ou à caryotype équilibré. Cependant, on observe aussi des spermatozoïdes déséquilibrés (par exemple disomie 13) et ce avec une fréquence non négligeable ( $8 \%$ dans notre étude, Pellestor et al, 1987).

Nous avons étudié dans le laboratoire 4 sujets porteurs de translocations réciproques (Pellestor et al, 1988, 1989). L'observation d'un nombre significatif de spermatozoïdes permet d'enregistrer différentes variétés de ségrégation et d'estimer leur fréquence. Deux d'entre elles prédominent largement chez tous les sujets : ce sont les ségrégations dites alternes et adjacentes 1. Or on sait depuis longtemps qu'au niveau de la descendance chaque translocation présente une prédisposition à un type donné de ségrégation. Ceci ne se trouve absolument pas vérifié au niveau des spermatozoïdes : quelle que soit la translocation, la distribution des ségrégations est toujours la même. L'observation des descendances avait conduit à supposer que chaque translocation avait son comportement propre, mais l'analyse des spermatozoïdes démontre l'existence d'une régulation non imputable à la méiose spermatocytaire, qui, pour sa part, donne toujours le même résultat. On est donc conduit à admettre que la régulation dépend exclusivement de l'interaction entre l'œuf fécondé anormal et l'environnement maternel (Sèle et al, 1989).

La confrontation des données cytogénétiques méiotiques et gamétiques chez le même sujet porteur de translocation serait du plus grand intérêt pour une meilleure compréhension de ces phénomènes. $\mathrm{Ce}$ n'est malheureusement possible qu'en théorie : l'étude gamétique exige un sperme fécondant, alors que l'étude méiotique implique une biopsie testiculaire a priori acceptée seulement dans le contexte d'une exploration d'infertilité masculine.

\section{RÉFÉRENCES}

Bongso A, Chye $\mathrm{Ng} \mathrm{S}$, Ratnam S, Sathananthan $H$, Wong PC (1988) Chromosome anomalies in human oocytes failing to fertilize after insemination in vitro. Hum Reprod 3, 645-649

Djalali $M$, Rosenbusch $B$, Wolf $M$, Sterzik $K$ (1988) Cytogenetics of unfertilized human oocytes. J Reprod Fertil 84, 647-649

Hassold T, Chiu D (1985) Maternal age-specific rates of numerical chromosome abnormalities with special reference to trisomy. Hum Genet 70, 11-17

Jagiello G, Karnicki J, Ryan RJ (1968) Superovulation with pituitary gonadotrophins: method for obtaining meiotic metaphase figures in human ova. Lancet, 178-180

Pellestor F, Sèle B (1988) Assessment of aneuploidy in the human female by using cytogenetics of IVF failures. Am J Hum Genet 42, 274-283

Pellestor F, Sèle B (1989) Etude cytogénétique du sperme humain. MS - Med Sci 5, 244-251

Pellestor F, Sèle B, Jalbert H (1987) Chromosome analysis of spermatozoa from a male heterozygous for a 13-14 robertsonian translocation. Hum Genet 76, 116-120

Pellestor F, Sèle B, Jalbert P (1988) Caryotypes de spermatozoìdes et ségrégations méiotiques. Etude de 4 translocations réciproques. $J$ Génét Hum 36, 103-106

Pellestor F, Sèle B, Jalbert H, Jalbert P (1989) Direct segregation analysis of reciprocal translocations: a study of $\mathbf{2 8 3}$ sperm karyotypes from four carriers. Am J Hum Genet 44, 464-473

Plachot M, Veiga A, Montagut J, de Grouchy J, Calderon G, Leprêtre S, Junca AM, Santalo J, Carles E, Mandelbaum J, Barri P, Degoy J, Cohen J, Egozcue J, Sabatier JC, SalatBaroux J (1988) Are clinical and biological IVF parameters correlated with chromosomal disorders in early life: a multicentric study. Hum Reprod 3, 627-635

Sèle B, Pellestor F, Jalbert P, Estrade C, Ostorero $C$, Gelas $M$ (1985) Analyse cytogénétique des pronucléus à partir du modèle hétérospécifique homme-hamster. Ann Génét $28,81-85$

Sèle B, Pellestor F, Chouteau J (1987) Influence de l'âge sur les anomalies chromosomiques de l'ovocyte humain. Etude de 124 caryo- 
types d'ovocytes provenant d'échecs de FIV. Contracept Fertil Sex 15, 786-788

Sèle B, Pellestor F, Jalbert $P(1989)$ Behaviour of reciprocal translocations: a new model deduced from carriers sperm cytogenetics. $A m$ $J$ Hum Genet 43, suppl A 121

Van Blerkom J, Henry G (1988) Cytogenetic analysis of living human oocytes: cellular ba- sis and developmental consequences of perturbations in chromosomal organization and complement. Hum Reprod 3, 777-790

Veiga A, Calderon G, Santalo J, Barri PN, Egozcue $J$ (1987) Chromosome studies in oocytes and zygotes from an IVF program. Hum Reprod $2,425-430$ 